\title{
TENDÊNCIA SECULAR DE CRESCIMENTO EM ESCOLARES DE PAULÍNIA, SÃO PAULO-BRASIL (1979/80 - 1993/94)
}

\author{
* Denise Barbieri Marmo, Mariana Porto Zambon, André Moreno Morcillo, luis Manuel Guimarey \\ Trabalho realizado no Departamento de Pediatria da Faculdade de Ciências Médicas da Unicamp, Campinas, SP
}

RESUMO - OBJETIVO. Avaliar a tendência secular de altura, peso e da relação peso/altura em escolares de Paulínia, São Paulo, Brasil, entre 1979/80 e 1993/94.

Métodos. Medidas antropométricas de peso $(P)$ e altura $(A)$ e da relação $\mathrm{P} / \mathrm{A}$ de 1.903 crianças de 6,5 a 12,5 anos ( $51,5 \% \mathrm{M}$ e $48,5 \% \mathrm{~F})$ de escolas públicas de Paulínia foram comparadas com dados de estudo anterior realizado na mesma cidade, com intervalo de 15 anos. Calculou-se o incremento por década e alisou-se os dados pela técnica de médias e medianas móveis $(3 \mathrm{H} 3 \mathrm{H} 3)$.

Resultados. As médias de altura e peso foram sempre superiores às do estudo anterior, com incrementos positivos. Os incrementos para a altura variaram de I, 13 a $5 \mathrm{~cm}$ no sexo masculino e I,2 a $4,33 \mathrm{~cm}$ nas meninas, já para o peso a variação foi de 0,53 a 4,13 kg nos meninos e 0,87 a $3,0 \mathrm{~kg}$ nas meninas. Para a relaçáo $P / A$, as médias dos dois estudos foram muito semelhantes em ambos os sexos.

CoNCLUsóes. A tendência secular positiva da estatura e do peso neste período deve-se principalmente ao desenvolvimento econômico, de saneamento básico, de habitação, de educação e na área de saúde ocorrido na cidade de Paulínia. A tendência secular positiva encontrada foi semelhante à observada em países desenvolvidos após a II Guerra Mundial e de outros trabalhos brasileiros.

Unitermos: Tendência secular. Crescimento. Desenvolvimento.

\section{INTRODUÇÃo}

Tendência secular refere-se a qualquer mudança do tamanho corporal ou de sua composição em determinado grupo populacional em longos períodos de tempo ${ }^{1,2}$. Nas últimas décadas o estudo da tendência seculartem sido motivo de grande interesse para os auxologistas. Estas mudanças, quando positivas, não estão associadas basicamente a fatores genéticos, mas sim a fatos sociais como a normatização do trabalho infantil, ao tamanho das famílias, às alterações do comportamento infantil e às condições de saúde individual e populacional $1^{3,4}$.

Em relação à altura, a tendência secular positiva ocorreu em vários grupos sociais e econômicos na Europa, Japão e nos Estados Unidos no século XX, principalmente após a II Guerra Mundial ${ }^{2,5-8}$. As estimativas do aumento da estatura variam com a idade e com o nível social e econômico de grupos populacionais de cada país.

Aproximadamente desde 1918 não ocorre mais aumento estatural nas classes sociais mais altas dos Estados Unidos². A tendência

\section{*Correspondência:}

Av. Jesuíno Marcondes Machado, 2201 - apto. 52C Chácara da Barra - CEP: 13092-321 - Campinas - SP

Fax: (55-19) 3788-826 - mlzambon@uol.com.br secularpositiva de estatura também cessou em Londres, entre os anos de 1959 e 1966, e no Japão entre 1970 e 1975'. Em vários outros países europeus, assim como em países em desenvolvimento, entretanto, a estatura continua a aumentar.

É interessante a constatação de que, mesmo nos países subdesenvolvidos e em desenvolvimento, em trabalhos realizados com grupos populacionais mais favorecidos, a tendência secular se mostra positiva, em contraste com o que ocorre com o restante do país ${ }^{10}$. No México, em crianças de nível social e econômico médio, uma avaliação da estatura realizada em 1970, comparada ao ano de 1920, mostrou diferenças significativas".

No Brasil, são poucos os estudos sobre tendência secular de crescimento e todos demonstram uma tendência positiva, principalmente nas classes sociais mais privilegiadas ${ }^{12-15}$.

Este estudo tem como objetivo avaliar a presença de tendência secular de altura, peso e da relação peso/altura em uma população de escolares, na faixa etária de 6,5 a I2,5 anos, da cidade de Paulínia, São Paulo, entre os períodos de 1979/80 e 1993/94.

\section{Métodos}

De um total de 12 escolas públicas existentes na cidade de Paulínia, interior de São Paulo, foram avaliadas 1.903 crianças entre 6,5 a 12,5 anos, 978 meninos (51,5\%) e 925 meninas (48,5\%), ou seja, $75 \%$ de crianças matriculadas nas quatro primeiras séries do primeiro grau de sete escolas pertencentes ao Programa de Saúde Escolar (PIESE) da cidade. Os autores avaliavam estas crianças como parte de seu trabalho de rotina e estas escolas não foram escolhidas nem sorteadas; eram aquelas determinadas para avaliação neste período.

Todas as crianças foram submetidas a um exame antropométrico, realizado por um único observador, entre março de 1993 e novembro de 1994, incluindo as medidas de peso (P) e altura (A). A altura foi medida com um antropômetro de madeira (precisão 0, I cm) e o peso em uma balança mecânica (precisão $100 \mathrm{~g}$ ).

Estes foram comparados aos de outro estudo realizado previamente na mesma cidade, com 8 I 2 estudantes, também de escolas públicas, com avaliação antropométrica pela mesma metodologia, de faixa etária comparativa. Portanto, as crianças foram agrupadas por idade, em intervalos de seis meses, de forma semeIhante ao estudo anterior ${ }^{16}$. Foram determinados as médias e os desvios padrão para a altura, o peso e a relação peso-altura (P/A). Os incrementos por década foram calculados entre os anos de 1979 e 1994. Foram calculados os intervalos de confiança (95\%) para peso e altura. 
Para o alisamento dos dados, de ambos os estudos, foi utilizada a técnica de Médias e Medianas Móveis $(3 \mathrm{H} 3 \mathrm{H} 3)^{17}$.

\section{Resultados}

São apresentados os valores da média e do desvio padrão da altura para o sexo masculino e feminino (Tabela I) e do peso (Tabela 2) dos estudos de 1979/80 e 1993/94, assim como o incremento por década, para cada faixa etária (Figuras I e 2). Os intervalos de confiança (95\%) encontram-se na Tabela 3.

As médias da altura no estudo de 1993/94 foram sempre superiores às de 1979/80. Os incrementos variaram de I, I $3 \mathrm{~cm}$ aos I I anos a $5 \mathrm{~cm}$ aos 12 anos no sexo masculino, e de I,20 cm aos oito anos a 4,33cm aos 6,5 anos para o sexo feminino.

As médias do peso do estudo de 1993/94 foram sempre superiores às de 1979/80. Os incrementos variaram de 0,53 kg aos I I anos a 4, $13 \mathrm{~kg}$ aos 12 anos no sexo masculino, e de 0,87 kg aos | |,5 anos a $3 \mathrm{~kg}$ aos I I anos para ao sexo feminino.

$\mathrm{Na}$ Tabela 4 são apresentados, para o sexo masculino e feminino, os valores da média e do desvio padrão da relação peso/altura do estudo de 1993/94 e somente o valor da média para o estudo de 1979/80, além do incremento por década. Em ambos os sexos, as médias dos dois estudos foram muito semeIhantes, com exceção das medidas de $145 \mathrm{~cm}$ $(2,13 \mathrm{~cm}$ de incremento) e $155 \mathrm{~cm}(3,33 \mathrm{~cm}$ de incremento) nos meninos.

\section{Discussão}

Paulínia tornou-se município em 1964 e, nas últimas décadas, apresentou desenvolvimento acentuado transformando sua base econômica de agrícola em industrial. Este processo teve início a partir de 1942 com a instalação da Rhodia S.A. e da Refinaria do Planalto (REPLAN) em 1964, dando origem a um importante pólo petroquímico. Concomitante a este progresso, ocorreu significativo crescimento populacional e aumento na taxa de urbanização, que foi acompanhado de importantes adaptações nas áreas de saneamento básico, habitação, educação e saúde ${ }^{18,19}$.

Com esta estrutura, a cidade apresentava na época cobertura vacinal de $90 \%$ e coeficiente de mortalidade infantil inferior ao da região sudeste desde 1975'9. Estudos com

\begin{tabular}{|c|c|c|c|c|c|c|c|c|}
\hline \multirow{4}{*}{$\begin{array}{l}\text { Idade } \\
\text { (anos) }\end{array}$} & \multicolumn{8}{|c|}{$\begin{array}{l}\text { Tabela I - Média e desvio padrão (DP) de estatura (cm) e peso (kg) e seus incrementos } \\
\text { por década de meninos de Paulínia (SP) em dois períodos (1979/80 - 1993/94) }\end{array}$} \\
\hline & \multicolumn{3}{|c|}{$1979 / 80$} & \multicolumn{3}{|c|}{$1993 / 94$} & \multicolumn{2}{|c|}{ Incremento } \\
\hline & & altura & peso & & altura & peso & Altura & Peso \\
\hline & $\mathrm{N}$ & Média(DP) & Média(DP) & $\mathbf{N}$ & $\overline{\text { Média(DP) }}$ & $\overline{\text { Média(DP) }}$ & (cm) & $(\mathbf{k g})$ \\
\hline 6,5 & $\overline{6}$ & || $4, \mid(6,0)$ & $\overline{19,8(2,81)}$ & $\overline{75}$ & $\overline{|2|, \mid(5,44)}$ & $\overline{23,2(3,84)}$ & 4,67 & 2,27 \\
\hline 7,0 & 32 & $117,4(6,0)$ & $21,2(3,16)$ & 199 & $123,2(5,42)$ & $24,1(4,67)$ & 3,87 & 1,93 \\
\hline 7,5 & 36 & $120,9(6,0)$ & $22,7(3,55)$ & 137 & $125,7(5,48)$ & $25,0(4,97)$ & 3,20 & 1,53 \\
\hline 8,0 & 35 & $124,0(5,9)$ & $24,0(3,98)$ & 49 & $127,6(6, \mid 1)$ & $26,5(5,99)$ & 2,40 & 1,67 \\
\hline 8,5 & 31 & $126,9(5,8)$ & $25,3(4,34)$ & 48 & $130,7(6,60)$ & $27,1(4,47)$ & 2,53 & 1,20 \\
\hline 9,0 & 34 & $129,5(5,8)$ & $26,6(4,60)$ & 92 & $133,4(6,65)$ & $30,0(6,13)$ & 2,60 & 2,27 \\
\hline 9,5 & 39 & $132,0(6,0)$ & $27,8(4,94)$ & 107 & $\mid 35,0(5,87)$ & $30,2(5,50)$ & 2,00 & 1,60 \\
\hline 10,0 & 44 & $137,0(6,5)$ & $29,1(5,35)$ & 81 & $139,4(6,37)$ & $33,6(7,81)$ & 1,60 & 3,00 \\
\hline 10,5 & 40 & $137,0(6,5)$ & $30,8(5,55)$ & 80 & $140,6(6,92)$ & $34,8(8,19)$ & 2,40 & 2,67 \\
\hline $\mid 1,0$ & 27 & $139, \mid(6,7)$ & $32,4(5,55)$ & 44 & $\mid 40,8(7,37)$ & $33,2(6,87)$ & 1,13 & 0,53 \\
\hline 11,5 & 38 & $140,2(6,8)$ & $33,5(5,44)$ & 33 & $\mid 43,7(6,97)$ & $37,8(9,10)$ & 2,33 & 2,87 \\
\hline 12,0 & 38 & $\mid 40,6(7,0)$ & $34,2(5,44)$ & 33 & $|48|,(8,17)$ & $40,4(9,67)$ & 5,00 & 4,13 \\
\hline
\end{tabular}

Tabela 2 - Média e desvio padrão (DP) de estatura (cm) e peso (kg) e seus incrementos por década de meninas de Paulínia (SP) em dois períodos (1979/80 - 1993/94)

\begin{tabular}{|c|c|c|c|c|c|c|c|c|}
\hline \multirow{3}{*}{$\begin{array}{l}\text { Idade } \\
\text { (anos) }\end{array}$} & \multicolumn{3}{|c|}{$1979 / 80$} & \multicolumn{3}{|c|}{ 1993/94 } & \multicolumn{2}{|c|}{ Incremento } \\
\hline & & altura & peso & & altura & peso & Altura & Peso \\
\hline & $\mathrm{N}$ & Média(DP) & Média(DP) & $\mathbf{N}$ & $\overline{\text { Média(DP) }}$ & $\overline{\text { Média(DP) }}$ & $\overline{(\mathrm{cm})}$ & $\overline{(\mathrm{kg})}$ \\
\hline 6,5 & $\overline{11}$ & $1 \mid 3,7(6,9)$ & $19,9(3,17)$ & $\overline{68}$ & $\overline{120,2(5,03)}$ & $23,1(5,26)$ & 4,33 & $\overline{2,13}$ \\
\hline 7,0 & 19 & | 17,2(6,9) & $21,4(3,66)$ & 178 & $122,7(5,68)$ & $24, I(4,88)$ & 3,67 & 1,80 \\
\hline 7,5 & 35 & $121,0(6,9)$ & $22,9(4,21)$ & 136 & $\mid 24,4(5,9 \mid)$ & $25,0(5,91)$ & 2,27 & 1,40 \\
\hline 8,0 & 40 & $124,3(6,7)$ & $24,3(4,58)$ & 40 & $126,1(6,97)$ & $25,8(5,55)$ & 1,20 & 1,00 \\
\hline 8,5 & 35 & $127,2(6,4)$ & $25,7(4,79)$ & 57 & $130,4(6,46)$ & $27,8(5,50)$ & 2,13 & 1,40 \\
\hline 9,0 & 35 & $129,7(6,1)$ & $27,0(5,13)$ & 98 & $132,9(6,64)$ & $29,4(6,09)$ & 2,13 & 1,60 \\
\hline 9,5 & 46 & $|32|,(6,1)$ & $28,5(5,7$ & 116 & $134,8(6,29)$ & $30,0(6,23)$ & 1,80 & 1,00 \\
\hline 10,0 & 42 & $134,6(6,4)$ & $30,2(6,57)$ & 88 & $139,5(6,28)$ & $33,4(7,72)$ & 3,27 & 2,13 \\
\hline 10,5 & 32 & $137,0(6,9)$ & $31,7(7,40)$ & 58 & $\mid 43,0(7,44)$ & $35,3(8,79)$ & 4,00 & 2,40 \\
\hline $\mid 1,0$ & 39 & $139,6(7,2)$ & $33,4(8,23)$ & 40 & $\mid 44,6(9,48)$ & $37,9(9,64)$ & 3,33 & 3,00 \\
\hline 11,5 & 42 & $\mid 43,0(7,4)$ & $35,8(8,91)$ & 29 & $\mid 46,3(6,48)$ & $37, I(7,45)$ & 2,20 & 0,87 \\
\hline 12,0 & 36 & $\mid 46,9(7,4)$ & $38,8(9,41)$ & 17 & $\mid 49,3(7,99)$ & $43,1(7,89)$ & 1,60 & 2,87 \\
\hline
\end{tabular}

crianças de pré-escolas e creches municipais da cidade de Paulínia demonstram uma condição nutricional privilegiada em relação ao nosso país 20,21 .

No estudo, atual foi possível verificar que, em todas as faixas etárias estudadas e em ambos os sexos, os valores das médias de altura e de peso foram superiores aos do estudo de Guimarey (1983) 16, demonstrando uma tendência secular positiva para este grupo populacional. Os incrementos de estatura foram de até $5 \mathrm{~cm} /$ década aos 12 anos para o sexo masculino, sendo para o feminino de até $4,3 \mathrm{~cm} /$ década aos 6,5 anos de idade. Os incrementos ponderais também foram significativos, sendo de 4, I kg/década no sexo masculino aos 12 anos e de $3 \mathrm{~kg} /$ década para o sexo feminino aos I I anos. Por outro lado, na avaliação da relação peso/altura não houve modificações.

Trabalhos realizados em países desenvolvidos como Estados Unidos, Canadá, Japão e Noruega sugerem que, entre os anos de 1860 e 1960, ocorreu aumento estatural de 1,3 cm 
MARMO DB ET AL.

Figura I - Distribuição dos incrementos por década de altura (cm) para o sexo masculino (-) e para o feminino (---) em escolares da cidade de Paulínia, São Paulo, referentes ao período entre 1979/80 e 1993/94

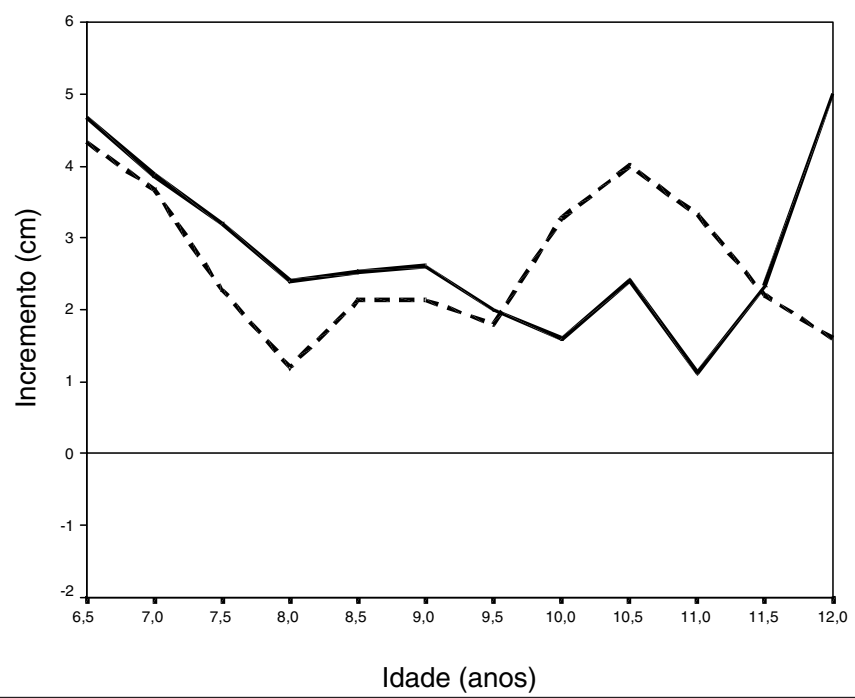

Figura2-Distribuição dos incrementos por década de peso (kg) para o sexo masculino (-) e para o feminino (....) em escolares da cidade de Paulínia, São Paulo, referentes ao período entre 1979/80 e 1993/94

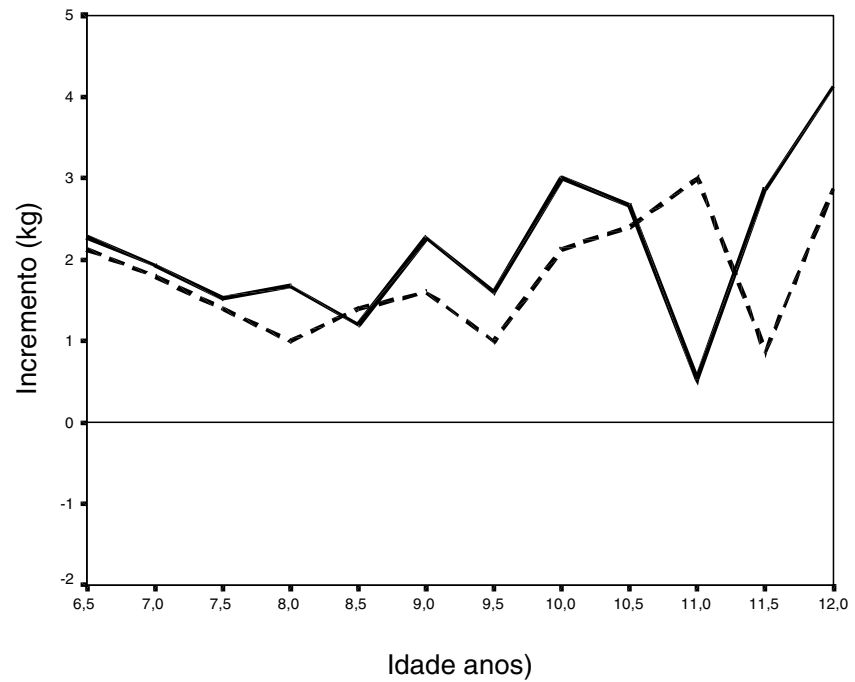

por década na infância (meninas de 8 anos e meninos de 10 anos), 1,9 cm/década na fase intermediária da adolescência (meninas aos 12 e meninos aos 14 anos) e $0,6 \mathrm{~cm} /$ década na idade adulta?.

Tanner $(|98|)^{22}$ também demonstra taxa de mudança secular estatural na Europa e América do Norte entre 1880 e $1950 \mathrm{de} \mathrm{Icm/}$ década entre os cinco e sete anos, $2,5 \mathrm{~cm} /$ década na adolescência e $1 \mathrm{~cm} /$ década nos adultos. No Japão, no período de 1900 a
1940, observou-se aumento de menos de $1 \mathrm{~cm} /$ década dos cinco aos sete anos, sendo que entre os 7 e 12 anos este foi de 3 a $5 \mathrm{~cm} /$ década entre os anos de 1950 e 197023.

Estudo mais recente com crianças de origem mexicana do sul do Texas, de nível socioeconômico baixo, nos anos de 1928 , 1972 e 1983, demonstrou ganho estatural nas idades de 7 a 14 anos, entre 1972 a 1983 , tão importante quanto o do período anterior (1928 a 1972$)^{24}$.
No Brasil, Koifman $(1987)^{12}$, estudando crianças de um município carioca entre 1960 e 1970, observou tendência secular positiva, assim como diferença estatural entre as classes econômicas, sendo os maiores valores nas classes privilegiadas. Monteiro et al. $(1994)^{13}$ demonstraram tendência secular de crescimento desde a metade do século XX, com aceleração nas últimas décadas, porém, ainda sem alcançar a expressão completa da sua genética estatural. De acordo com estes autores, o aumento na altura média dos brasileiros nascidos em 1967 em relação aos nascidos em 1952 foi de $0,8 \mathrm{~cm}$ por década para o sexo masculino e 0,6 cm por década para o feminino. Aos sete anos de idade, esta diferença foi maior, $3,6 \mathrm{~cm}$ para os meninos e 3,7 cm para as meninas. Em todas as regiões brasileiras e em ambos os sexos, a tendência secular de crescimento estatural foi francamente positiva, embora ainda ocorra uma distância significativa entre as alturas dos brasileiros das regiões Nordeste e Norte em comparação aos dados de referência (NCHS).

Espin Neto $(2001)^{14}$ em estudo retrospectivo realizado em colégio de classe social alta, da cidade do Rio de Janeiro, também demonstra tendência secular positiva. Estudo mais recente ${ }^{15} \mathrm{com}$ dados de 1998, realizado com escolares de classe média e alta da cidade de São Paulo de descendência européia, encontrou tendência positiva de crescimento em peso e estatura, assim como valores comparáveis ou até superiores aos de referência do NCHS (National Center for Health Statistics).

Em relação ao peso, existem menos trabalhos e acredita-se que seu incremento seja relativo ao aumento da estatura. Meredith $(1963)^{5}$, analisando dados norte-americanos de meninos de I a 20 anos no período de 1880 a 1960, demonstrou incrementos consideráveis, chegando a I,8k g por década.

$\mathrm{Na}$ Inglaterra, no período de $191 \mid \mathrm{a}$ $1954^{4}$, a taxa de incremento de peso por década foi de $0,2 \mathrm{~kg}$ aos cinco anos de idade, $0,8 \mathrm{~kg}$ aos nove anos e I,6 kg aos 13 anos, em ambos os sexos. Outros estudos realizados em vários países europeus demonstraram resultados semelhantes ou até o não incremento de peso, verificado nas crianças de seis anos de idade em Ontário, no período de 1923-19692. 
Tabela 3 - Intervalo de confiança $(95 \%)$ para altura $(\mathrm{cm})$ e peso $(\mathrm{kg})$ para os sexos masculino e feminino nos dois períodos $1979 / 80$ e 1993/94

\begin{tabular}{|c|c|c|c|c|c|c|c|c|}
\hline \multirow[t]{2}{*}{$\begin{array}{l}\text { Idade } \\
\text { (anos) }\end{array}$} & \multicolumn{2}{|c|}{$\begin{array}{l}\text { Altura masculina } \\
(\mathrm{cm})\end{array}$} & \multicolumn{2}{|c|}{$\begin{array}{l}\text { Altura feminina } \\
\qquad(\mathrm{cm})\end{array}$} & \multicolumn{2}{|c|}{$\begin{array}{l}\text { Peso masculino } \\
(\mathbf{k g})\end{array}$} & \multicolumn{2}{|c|}{$\begin{array}{l}\text { Peso feminino } \\
(\mathbf{k g})\end{array}$} \\
\hline & $1979 / 80$ & $1993 / 94$ & $1979 / 80$ & $1993 / 94$ & $1979 / 80$ & $1993 / 94$ & $1979 / 80$ & $1993 / 94$ \\
\hline 6,5 & $\begin{array}{l}109,30- \\
118,90\end{array}$ & $\begin{array}{l}119,86- \\
122,43\end{array}$ & $\begin{array}{l}109,62- \\
117,78\end{array}$ & $\begin{array}{l}119,00 \\
121,39\end{array}$ & $\begin{array}{l}17,55- \\
22,05\end{array}$ & $\begin{array}{l}22,33- \\
24,06\end{array}$ & $\begin{array}{l}18,02- \\
21,78\end{array}$ & $\begin{array}{l}21,84- \\
24,35\end{array}$ \\
\hline 7,0 & $\begin{array}{l}\text { ||15,32- } \\
|| 19,48\end{array}$ & $\begin{array}{l}122,45- \\
123,94\end{array}$ & $\begin{array}{l}114,10 \\
120,30\end{array}$ & $\begin{array}{l}121,86- \\
123,54\end{array}$ & $\begin{array}{l}20,10- \\
22,30\end{array}$ & $\begin{array}{l}23,45- \\
24,75\end{array}$ & $\begin{array}{l}19,75- \\
23,05\end{array}$ & $\begin{array}{l}23,37- \\
24,82\end{array}$ \\
\hline 7,5 & $\begin{array}{l}118,94- \\
122,86\end{array}$ & $\begin{array}{l}124,78- \\
126,62\end{array}$ & $\begin{array}{l}118,82- \\
123,18\end{array}$ & $\begin{array}{r}123,40 \\
125,40\end{array}$ & $\begin{array}{l}21,54- \\
23,86\end{array}$ & $\begin{array}{l}24,18- \\
25,82\end{array}$ & $\begin{array}{l}21,51- \\
24,29\end{array}$ & $\begin{array}{l}24,00- \\
26,00\end{array}$ \\
\hline 8,0 & $\begin{array}{l}122,04- \\
125,96\end{array}$ & $\begin{array}{r}125,89 \\
129,30\end{array}$ & $\begin{array}{l}122,22- \\
126,38\end{array}$ & $\begin{array}{l}123,95- \\
128,26\end{array}$ & $\begin{array}{l}22,69- \\
25,31\end{array}$ & $\begin{array}{l}24,81- \\
28,18\end{array}$ & $\begin{array}{l}22,89- \\
25,71\end{array}$ & $\begin{array}{l}24,07- \\
27,52\end{array}$ \\
\hline 8,5 & $\begin{array}{l}124,86- \\
128,94\end{array}$ & $\begin{array}{l}128,83- \\
132,56\end{array}$ & $\begin{array}{l}125,08- \\
129,32\end{array}$ & $\begin{array}{l}|28,7|- \\
\mid 32,08\end{array}$ & $\begin{array}{l}23,77- \\
26,83\end{array}$ & $\begin{array}{l}25,83- \\
28,37\end{array}$ & $\begin{array}{l}24,11- \\
27,29\end{array}$ & $\begin{array}{l}26,37- \\
29,23\end{array}$ \\
\hline 9,0 & $\begin{array}{l}\mid 27,56- \\
|3|, 44\end{array}$ & $\begin{array}{l}\mid 32,05- \\
\mid 34,75\end{array}$ & $\begin{array}{l}127,68- \\
|3|, 72\end{array}$ & $\begin{array}{l}|3|, 59- \\
|34,2|\end{array}$ & $\begin{array}{l}25,05- \\
28,15\end{array}$ & $\begin{array}{l}28,75- \\
31,25\end{array}$ & $\begin{array}{l}25,29 \\
28,70\end{array}$ & $\begin{array}{l}28,18- \\
30,61\end{array}$ \\
\hline 9,5 & $\begin{array}{l}130,12- \\
133,88\end{array}$ & $\begin{array}{l}133,88- \\
136,12\end{array}$ & $\begin{array}{l}130,34- \\
133,86\end{array}$ & $\begin{array}{l}133,66- \\
135,94\end{array}$ & $\begin{array}{l}26,25- \\
29,35\end{array}$ & $\begin{array}{l}29,16- \\
31,24\end{array}$ & $\begin{array}{l}26,83- \\
30,17\end{array}$ & $\begin{array}{l}28,86- \\
31,14\end{array}$ \\
\hline 10,0 & $\begin{array}{l}135,08- \\
138,92\end{array}$ & $\begin{array}{l}|38,0|- \\
\mid 40,79\end{array}$ & $\begin{array}{l}132,66- \\
136,54\end{array}$ & $\begin{array}{l}\mid 38,19 \\
|40,8|\end{array}$ & $\begin{array}{l}27,51- \\
30,69\end{array}$ & $\begin{array}{l}31,89 \\
35,30\end{array}$ & $\begin{array}{l}28,22- \\
32,18\end{array}$ & $\begin{array}{l}31,79 \\
35,01\end{array}$ \\
\hline 10,5 & $\begin{array}{l}134,98- \\
139,02\end{array}$ & $\begin{array}{l}\mid 39,09- \\
|42,1|\end{array}$ & $\begin{array}{l}|34,6|- \\
\mid 39,39\end{array}$ & $\begin{array}{l}|4|, 08- \\
\mid 44,92\end{array}$ & $\begin{array}{l}29,07- \\
32,52\end{array}$ & $\begin{array}{l}33,00- \\
36,60\end{array}$ & $\begin{array}{l}29,13- \\
34,27\end{array}$ & $\begin{array}{l}33,05- \\
37,55\end{array}$ \\
\hline 11,0 & $\begin{array}{l}\mid 36,57- \\
|4|, 63\end{array}$ & $\begin{array}{l}138,62- \\
\mid 42,97\end{array}$ & $\begin{array}{l}\mid 37,35- \\
|4|, 85\end{array}$ & $\begin{array}{l}|4|, 67- \\
\mid 47,54\end{array}$ & $\begin{array}{l}30,30- \\
34,50\end{array}$ & $\begin{array}{l}31,16- \\
35,24\end{array}$ & $\begin{array}{c}30,81- \\
35,99\end{array}$ & $\begin{array}{l}34,92- \\
40,88\end{array}$ \\
\hline 11,5 & $\begin{array}{l}138,04- \\
\mid 42,36\end{array}$ & $\begin{array}{l}|4|, 33- \\
\mid 46,07\end{array}$ & $\begin{array}{l}140,76- \\
\mid 45,23\end{array}$ & $\begin{array}{l}\mid 43,95- \\
\mid 48,65\end{array}$ & $\begin{array}{l}31,77- \\
35,22\end{array}$ & $\begin{array}{l}34,70- \\
40,90\end{array}$ & $\begin{array}{l}33,11- \\
38,48\end{array}$ & $\begin{array}{l}34,39- \\
39,80\end{array}$ \\
\hline 12,0 & $\begin{array}{l}138,36- \\
142,83\end{array}$ & $\begin{array}{l}\mid 45,32- \\
\mid 50,88\end{array}$ & $\begin{array}{c}\mid 44,49- \\
|49,3|\end{array}$ & $\begin{array}{l}145,50 \\
153,10\end{array}$ & $\begin{array}{l}32,47- \\
35,92\end{array}$ & $\begin{array}{c}37,11- \\
43,69\end{array}$ & $\begin{array}{r}35,72- \\
41,88\end{array}$ & $\begin{array}{c}39,36- \\
46,84\end{array}$ \\
\hline
\end{tabular}

\begin{tabular}{|c|c|c|c|c|c|c|c|c|c|c|}
\hline \multicolumn{11}{|c|}{$\begin{array}{l}\text { Tabela } 4 \text { - Média e desvio padrão (DP) de 1993/94 e média de 1979/80 da relação peso/altura (kg/m) e do } \\
\text { incremento por década em escolares de ambos os sexos da cidade de Paulínia - São Paulo }\end{array}$} \\
\hline \multirow{3}{*}{ Altura } & \multicolumn{5}{|c|}{ Sexo masculino } & \multicolumn{5}{|c|}{ Sexo feminino } \\
\hline & \multirow{2}{*}{$\begin{array}{l}\overline{1979 / 80} \\
\overline{\text { Média }}\end{array}$} & \multicolumn{3}{|c|}{ 1993/1994 } & \multirow{2}{*}{$\begin{array}{c}\text { Incremento } \\
\mathrm{cm}\end{array}$} & \multirow{2}{*}{$\begin{array}{l}979 / 1980 \\
\text { Média }\end{array}$} & \multicolumn{3}{|c|}{$1993 / 1994$} & \multirow{2}{*}{$\begin{array}{c}\text { Incremento } \\
\mathrm{cm}\end{array}$} \\
\hline & & $\overline{\mathbf{N}}$ & Média & $\overline{D P}$ & & & $\overline{\mathbf{N}}$ & Média & $\overline{D P}$ & \\
\hline 115 & 20,2 & 51 & 20,0 & 1,88 & $-0,13$ & 20,2 & 67 & 20,1 & 2,42 & $-0,07$ \\
\hline 120 & 21,7 & 123 & 22,3 & 2,34 & 0,40 & 22,4 & 130 & 21,9 & 2,20 & $-0,33$ \\
\hline 125 & 23,9 & 204 & 24,1 & 2,47 & 0,13 & 24,9 & 178 & 25,2 & 4,04 & 0,20 \\
\hline 130 & 26,9 & 176 & 27,5 & 3,57 & 0,40 & 26,9 & 153 & 27,1 & 4,05 & 0,13 \\
\hline 135 & 30,2 & 149 & 30,4 & 4,77 & 0,13 & 30,5 & 146 & 30,2 & 4,72 & $-0,20$ \\
\hline 140 & 32,8 & 127 & 33,2 & 5,12 & 0,27 & 33,5 & 106 & 33,8 & 6,04 & 0,20 \\
\hline 145 & 35,0 & 84 & 38,2 & 6,31 & 2,13 & 37,0 & 69 & 37,9 & 7,14 & 0,60 \\
\hline 150 & 38,9 & 35 & 40,0 & 6,50 & 0,73 & 41,7 & 45 & 41,6 & 6,59 & $-0,07$ \\
\hline 155 & 42,4 & 14 & 47,4 & 10,34 & 3,33 & 45,0 & 16 & 44,9 & 5,09 & $-0,07$ \\
\hline
\end{tabular}

A relação peso/altura não demonstrou grandes alterações nesse período. Estes dados são comparáveis com a evolução descrita por Ramos Galván \& Ramos Rodríguez (1988) ${ }^{(25)}$ em crianças sob situação de estresse nutricional, quando dizem que fisicamente a criança adquire nanismo nutricional, diminuição do peso e da estatura com boa relação peso/altura, teoricamente sem desnutrição. Guimarey et al. ( 1995$)^{26}$ acreditam nessa evolução e classificam essa forma clínica de desnutrição como "compensada".

Os dados existentes em toda a literatura, incluindo os estudos nacionais, deixam inúmeros questionamentos relativos à tendência secular. A tendência positiva que vem ocorrendo em nosso país nas avaliações mais recentes com indivíduos adultos também vem acontecendo na faixa etária pediátrica, principalmente nos escolares, e provavelmente estão correlacionadas.

Sem dúvida, ocorreram na cidade de Paulínia mudanças sociais, econômicas, em seu nível de atenção à saúde, educação e prestação de serviços que pudessem justificar mudanças positivas na tendência secular de crescimento.

\section{Conclusão}

Neste período, na cidade de Paulínia, encontrou-se tendência secular positiva de estatura e peso, semelhante à observada em países desenvolvidos após a II Guerra Mundial e de outros trabalhos brasileiros. Isso pode ser explicado pelo desenvolvimento ocorrido nesta cidade em vários setores como o econômico, o de saneamento básico, o de habitação, o de educação e na área de saúde.

\section{Conflito de interesse: não há.}

\section{SUMMARY}

Secular trends of growth of schoolchildren from Paulínia, Säo Paulo - Brazil (1979/80 - 1993/94)

BACKGROUND. The purpose of this study was to evaluate the seculartrends in height, weight and weight/height of schoolchildren from the city of Paulinia, São Paulo, Brazil between 1979/80 and 1993/94.

METHODS. Anthropometric measurements (height, weight and weight/height) of 1,903 children (6.5-12.5y); $51.5 \% \mathrm{M:}$ 48.5\% F, from Paulinia publicschools were compared with data from a previous study carried out in the same city 15 years earlier. Decade increments were calculated and data was smoothed by the technique of means and medians (3H3H3).

RESULTS. Height and weight mean values were always greater than those of the previous study, with positive increments. Height increments ranged from 1.13 to $5.0 \mathrm{~cm}$ in boys and 
Marmo DB ET AL.

from 1.2 to $4.33 \mathrm{~cm}$ in girls. Weight increments ranged from 0.53 to $4.13 \mathrm{~kg}$ in males and from 0.87 to $3.0 \mathrm{~kg}$ in females. In the two studies, weight/height means were very similar for both genders.

CONCLUSIONs. Increments in height and weight during this period are an indicator of development on the economical and health levels. A positive seculartrend was also observed in developed countries after the $2^{\text {nd }}$ World War and in the Brazilian people. [Rev Assoc Med Bras 2004; 50(4): 386-90]

KEY WORDS: Secular trend. Growth. Development.

\section{RefERÊNCIAS}

I. Hauspie RC, Vercauteren M, Susanne C. Secular Changes in Growth. Horm. Res. 1996; 45:8-17.

2. Roche AF. Secular Trend in Human Growth, Maturation and Development. Monogr. Soc. Res. Chil. Dev. 1979; 44 (3-4): I- 120.

3. Bielicki T. Physical Growth as a Measure of the Economic Well-Being of Populations: The Twentieth Century. In: Falkner F \& Tanner JM. Human Growth, New York, Plenum Press, 1986. p.283-305.

4. Eveleth PB. Population Differences in Growth: Environmental and Genetic Factors. Human Growth, New York, Plenum Press, 1986. p.22I-239.

5. Meredith HV. Change in the Stature and Body Weight of North American Boys During the Last 80 Years. Adv. Child Develop. Beh. 1963; 69-107.

6. Kimura KA. Consideration Of The Secular Trend in Japanese for Height and Weight by a Graphic Method. Am. J. Phys. Anthropol. 1967;27: 89-94.
7. Meredith HV. Findings from Asia, Australia, Europe and North America on Secular Change in Mean Height of Children and Young Adult. Am J. Phys Anthropol. 1976;44: 31 5-326.

8. Van Wieringen, JC. Secular Growth Changes. In: Falkner F \& Tanner JM. Human Growth. New York, Plenum Press, p.307$331,1986$.

9. Cameron N. - The Growth of London Schoolchildren 1904-1966: An Analysis of Secular Trend and Intra-County Variation. Ann. Hum. Biol. 1979;6: 505-525.

10. Malina RM. Research On Secular Trends in Auxology. Anthropol. Anz. 1990; 48:209-227.

I I. Ramos Galván R. Análisis de dos Estudios de Peso e Talla Hechos com 50 Años de Diferencia en Niños de la Ciudad de México. Bol Med Hosp Infant. 1978;35:44 I -463.

12. Koifman F. Crescimento em Escolares na Região de Irajá, Rio de Janeiro. Décadas de Sessenta e Setenta. São Paulo, 1987. (Tese De Doutoramento, Apresentada À Faculdade De Ciências Médicas Da USP).

13. Monteiro CA, Benício MHD'a, Gouveia NC. Secular Trends in Brazil Over Three Decades. Ann. Human. Biol. 1994; 21: 381-390.

14. Espin Neto J. Tendência Secular do Crescimento de Escolares Brasileiros de Alto Nível Sócio Econômico. Campinas, 200 I. (Tese de Doutoramento, Faculdade de Ciências Médicas da Unicamp).

15. Castilho LV \& Lahr MM. Secular Trends in Growth Among Urban Brazilian Children of European Descent. Ann. Hum. Biol. 200I; 28:564-574.

16. Guimarey LM. Crescimento e Estado Nutricional em Escolares de PaulíniaSão Paulo-Brasil. Campinas, 1983. (Tese de Doutoramento, Faculdade de Ciências Médicas da Unicamp).

17. Tukey J W. Exploratory Data Analysis. Phillippines, Addison-Wesley, 1977.

18. Neves JLS. Desenvolvimento Econômico e Urbanização. Estudo de Caso do Município de Paulínia. Campinas, 1984. (Relatório Final de
Monografia Apresentada no Instituto de Economia da Unicamp).

19. Seade Fundação Sistema Estadual de Análise de Dados, - Http:/Nww.Seade.Gov.Br, 1999.

20. Zanolli ML. Avaliação do Estado Nutricional de Pré-Escolares Matriculados nas Escolas Municipais de Educação Infantil de Paulínia. Campinas, 1992. (Dissertação de Mestrado Apresentada à Faculdade de Ciências Médicas da Unicamp).

21. Antonio MARGM. Avaliação do Estado Nutricional e do Perfil de Crescimento de 568 Crianças Matriculadas nas 14 Creches Municipais de Paulínia - S.P. Campinas, 1995. (Dissertação de Mestrado Apresentada à Faculdade de Ciências Médicas da Unicamp).

22. Tanner JM. A History of the Study of Human Growth. Cambridge, Cambridge University Press, 1981. 499p.

23. Tanner JM, Hayashi T, Preece M.A, Cameron $\mathrm{N}$. Increase in Lenght of Leg Relative to Trunk in Japanese Children and Adults From 1957 to 1977: Comparison With British and With Japanese Americans. Ann. Hum. Biol. 1982; 9:4II-423.

24. Malina RM, Zavaleta NA, Little BB. Secular Changes in the Stature and Weight of Mexican American Schoolchildren in Brownsville, Texas, Between 1928 and 1983. Hum. Biol. 1987; 59:509-522.

25. Ramos Galván R \& Ramos Rodríguez RM. El Escolar. In: Cusminsky M, Moreno EM, Ojeda ENS. Crescimiento y Desarrollo - Hechos y Tendencias. Washington D.C., Organizacion Panamericana de la Salud, 1988. p295-323.

26. Guimarey LM, Carnese FR, Pucciarelli HM. La Influencia Ambiental en el Crecimiento Humano. Ciencia Hoy. 1995; 5:41-47.

Artigo recebido: 10/10/03 Aceito para publicação: 06/07/04 